\title{
artigo
}

Santos, A. R., Moura, V. L. L., Koller, F. J., Batista, J., Burdzinski, V. F., Oliveira, A. A. S.

Incidência de lesão por pressão em pacientes internados em um hospital com certificação de qualidade

\section{Incidência de lesão por pressão em pacientes internados em um hospital com certificação de qualidade}

\author{
Incidence of pressure injury in patients hospitalized in a hospital with quality certification \\ Incidencia de lesión por presión en pacientes hospitalizados en un hospital con certificación de calidad
}

\section{RESUMO}

Objetivo: Caracterizar os casos de participantes com lesão por pressão em hospital privado e acreditado com protocolo de prevenção instituído. Método: Estudo de caso múltiplo realizado em um hospital privado, a amostragem foi de 100 prontuários eletrônicos aleatórios mais 2 casos os quais foram notificados no setor de qualidade. Resultados: Foram subnotificados $28 \%$ dos incidentes sobre lesão por pressão, conforme dados obtidos do setor de qualidade da instituição hospitalar. 0 perfil dos participantes identificados nos prontuários corresponde ao gênero masculino, idosos, com doença crônica existente e com tempo de internação superior a cinco dias. Em relação à região do corpo com mais injúria, destacou-se a região sacra. Conclusão: Notou-se a importância da aplicabilidade do protocolo de prevenção de lesão por pressão e o alinhamento do conhecimento do enfermeiro acerca do assunto veste as mudanças das tecnologias em saúde.

DESCRITORES: Lesão por Pressão; Qualidade da Assistência à Saúde; Cuidados de Enfermagem; Avaliação em Enfermagem; Segurança do Paciente.

\section{ABSTRACT}

Objective: Characterizing the cases of participants with pressure injury in a private hospital and accredited with a prevention protocol instituted. Method: Amultiple case study conducted in a private hospital; the sampling was 100 random electronic medical records plus 2 cases that were reported in the quality sector. Results: Of incidents of pressure injury $28 \%$ were underreported, according to data obtained from the quality sector of the hospital institution. The profile of the participants identified in the medical records corresponds to the male gender, elderly, with existing chronic disease and with hospitalization time of more than five days. In relation to the region of the body with the most injury, the sacred region stood out. Conclusion: It was noted the importance of the applicability of the protocol for the prevention of pressure injury and the alignment of the nurse's knowledge about the subject, the changes in health technologies.

DESCRIPTORS: Pressure Injury; Quality of Health Care; Nursing Care; Nursing Evaluation; Patient Safety.

\section{RESUMEN}

Objetivo: Caracterizar los casos de participantes con lesión por presión en un hospital privado y acreditado con un protocolo de prevención establecido. Método: Unestudio de caso múltiple realizado en un hospital privado, el muestreo fue de 100 historias clínicas electrónicas aleatorias más 2 casos que se notificaron en el sector de calidad. Resultados: El 28\% de los incidentes de lesión por presión fueron subreportados según datos obtenidos del sector de calidad de la institución hospitalaria. El perfil de los participantes identificados en las historias clínicas corresponde al sexo masculino, ancianos, con enfermedad crónica existente y con tiempo de hospitalización de más de cinco días. En relación con la región del cuerpo con más lesiones, destacó la región sacra. Conclusión: Se observó la importancia de la aplicabilidad del protocolo para la prevención de la lesión por presión y la alineación de los conocimientos de la enfermera sobre el tema, los cambios en las tecnologías de salud.

DESCRIPTORES: Lesión por Presión; Calidad de la Atención de la salud; Cuidados de Enfermería; Evaluación de Enfermería; Seguridad del Paciente.

RECEBIDO EM: 08/06/21 APROVADO EM: 14/06/21

\section{Aline Renata dos Santos}

Acadêmica de Enfermagem do Centro Universitário Santa Cruz. ORCID: 0000-0002-8420-3643

\section{Vanessa Leal de Lima de Moura}

Acadêmica de Enfermagem do Centro Universitário Santa Cruz. ORCID: 0000-0002-8420-3643 


\section{Francisco José Koller}

Docente do Curso de Enfermagem do Centro Universitário Santa Cruz. Doutorado em Enfermagem pela UFPR. ORCID: 0000-0002-2911-7670

\section{Josemar Batista}

Docente do Curso de Enfermagem do Centro Universitário Santa Cruz. Mestre em Enfermagem pela UFPR. ORCID: 0000-0002-8420-3643

\section{Vanessa de Fátima Burdzinski}

Coordenadora da Rede Brasileira de Enfermagem e Segurança do Paciente Núcleo Curitiba-PR. Enfermeira pela PUCPR. ORCID: 0000-0003-3145-1683

\section{Adrieli Aparecida Simões de Oliveira}

Enfermeira Pós-graduada em Estomaterapia na Pontifícia Católica do Paraná. ORCID: 0000-0001-8052-7993

\section{INTRODUÇÃO}

A $s$ instituições de saúde visam qualificação os serviços prestados através de processos de certificação hospitalar nacional e internacional, com vista a atestar os serviços prestados na assistência ao paciente, na segurança ocupacional, utilização eficiente de recursos e gestão das informações para melhoria contínua, que pode ser manifestada na ocorrência de um evento adverso 1 .

O evento adverso está relacionado diretamente à segurança do paciente, a qual a Organização Mundial da Saúde (OMS), com o intuito de reduzir ao mínimo o risco ao que o paciente é submetido quando exposto a procedimentos vinculados ao tratamento de saúde2. Entende-se que quando ocorre implicação da estrutura ou função do corpo e/ou qualquer efeito proveniente de assistência à saúde, ocorreu-se comprometimento segurança do paciente3.

O Ministério da Saúde define que o evento adverso pode ser "incidente que causa danos à saúde" 4 , dentre estes agravos cita-se: erro na cadeia medicamentosa, cirurgia em paciente ou parte do corpo errada e a lesão por pressão (LP) desenvolvida em pacientes durante a internação e causa injúrias a saúde do indivíduo5.A incidência da LP está associada durante ao período hospitalar decorrente do cisalhamento e fricção da pele decorrente da deficiência nutricional, alteração circulação sanguínea, doenças crônicas, mobilidade comprome-

\section{O evento adverso} está relacionado diretamente à segurança do paciente, a qual

a Organização

Mundial da Saúde

(OMS), com o

intuito de reduzir

ao mínimo o risco

ao que o paciente

é submetido

quando exposto

a procedimentos

vinculados ao

tratamento de saúde tida, tempo de internação6, além de trazer danos à imagem da equipe assistencial, da instituição e aumento do custo financeiro7.

A formação de LP, além de causar um desconforto e sofrimento ao paciente, demonstra uma falha na execução do protocolo de prevenção e aumenta o custo deste paciente à instituição, visto que a implantação de medidas preventivas e seu dispêndio financeiro é menor se comparando com o custo para o tratamento, como exemplo a implementação da Escala de Braden que não tem custo financeiro à instituição e trata-se de uma ferramenta validada para uso pelo profissional enfermeiro8.

Em 2013, foi instituído no Brasil o Programa Nacional de Segurança do Paciente (PNSP), o qual objetivo é a prevenção de evento adverso que pode ocorrer com o paciente por falha na assistência. O PNSP traz protocolos primordiais à assistência multidisciplinares dentro da instituição, os quais abordam: higienização das mãos; identificação do paciente; risco de quedas; segurança na prescrição, uso e administração de medicamentos; prevenção de úlcera por pressão e cirurgia segura9.

É evidente a importância do conhecimento do enfermeiro em relação ao desenvolvimento da LP e cabe a ele a elaboração de um protocolo efetivo para a prevenção deste dano, com o objetivo de confeccionar uma ferramenta que auxilie a tomada de conduta assistencial que transmita detalhamento operacional, baseado em evidência cientifica que proporcione conhecimento, 


\section{artigo}

Santos, A. R., Moura, V. L. L., Koller, F. J., Batista, J., Burdzinski, V. F., Oliveira, A. A. S

Incidência de lesão por pressão em pacientes internados em um hospital com certificação de qualidade

propague uma comunicação assertiva e padronização do cuidado entre os profissionais resultando em uma assistência de qualidade10.

Um estudo documental de 566 pacientes que passaram pela UTI do Hospital Universitário do município de Petrolina-PE, evidenciou a efetividade do protocolo de prevenção de LP, após sua instituição constatou-se a redução de LP, a qual de $36,6 \%$ para $12,3 \%$ de pacientes acometidos por esse dano e houve redução no tempo de internação que foi de 11,4 dias para 7,1 dias11.

$\mathrm{Na}$ revisão de literatura com 19 artigos relacionados ao tema de gestão de saúde, destacou-se a alta incidência de LP no Brasil em relação aos países desenvolvidos, faz com que o PNSP dedicasse uma atenção a prevenção de LP12. E a OMS destaca a necessidade de utilizar indicadores que determinam a eficácia do serviço prestado, e revela que $95 \%$ das LP podem ser evitadas e salienta da importância de ações preventivas e tratamentos mais efetivos em lesões já estabelecidas 13

Dados levantados em um estudo desenvolvido em uma unidade hospitalar da Fundação de Minas Gerais ilustraram o peso da falta de prevenção ao custo financeiro, onde ele traz o acréscimo dos seguintes valores: $\mathrm{R} \$ 98,90$ a $\mathrm{R} \$ 180,00$ por dia na diária do internamento do paciente o valor aumenta conforme o estadiamento da lesão, trazendo esses os valores diários para mensal resulta em R\$915,75 a R\$36.929,95 e chegando a $\mathrm{R} \$ 445.664,38$ anualmente falando, sem considerar gastos com recursos humanos e físicos14. Com base no desprendimento financeiro, reduzir a incidência de LP com ações preventivas é a forma mais inteligente em evitar esse custo e dano ao paciente 12 .

O presente estudo tem como objetivo caracterizar os casos de pacientes com lesão por pressão, em hospital privado e acreditado internacionalmente emCuritiba - PR, Brasil.

\section{MÉTODO}

Trata se de um estudo de caso múltiplo, realizado no mês de novembro de 2020 , com total de 100 prontuários aleatórios referente ao período de internação de janeiro a setembro de 2020, em um hospital privado, com certificação de acreditação internacional com o título Qmentum International, localizado no município de Curitiba - PR, Brasil. Esse hospital é composto de 82 leitos, com prestação de serviço de atendimento de média e alta complexidade. Os critérios de inclusão utilizados foram: prontuários dos seguintes setores: unidade de terapia intensiva (UTI) e de internação, participantes com idade superior a 18 anos, de ambos os sexos, com patologias clínicas e cirúrgicas, com utilização da escala de Braden preenchida durante o período de internação, com tempo de internação igual ou superior a cinco dias. Os critérios de exclusão foram os prontuários com ausência ou falta de seguimento da Escala de Braden, participantes com lesão de pressão provindos de ambiente domiciliar, transferidos de outra instituição de saúde ou de longa permanência para idosos.

A coleta de dados foi realizada pelos pesquisadores com dupla análise dos pron-

\section{TABELA 1 - Perfil dos pacientes acometidos por LP no hospital privado de Curitiba-Pr}

$$
\text { CONDIÇÕES DO CASO E DA LP }
$$

Escala de Braden

Risco Brando (15 há 16)

n.

Risco Moderado (12 há 14)

4

Faixa etária

Abaixo de 59 anos

29

60 a 69 anos

14

71 a 79 anos

29

80 a 89 anos

29

Sexo

Feminino

Masculino

Escala de Glasgow

Moderado

14

Grave

29

Leve

Doença crônica

Sim

Não

Localização da LP

Sacra

Outras regiões 
LP. Do total da amostra analisada destacou-se Braden com risco brando (57\%; n $=4)$, idade superior a 60 anos $(87 \% ; \mathrm{n}=$ $5)$, sexo masculino $(71 \% ; n=5)$, Glasgow leve $(57 \% ; \mathrm{n}=4)$, pacientes acometidos por alguma doença crônica $(71 \% ; \mathrm{n}=5)$, região sacra ( $71 \% ; n=5)$, estágio I $(71 \%$; $\mathrm{n}=5)$, tempo de internação superior a seis dias $(86 \% ; n=6)$, única lesão $(57 \% ; n=4)$. Conforme evidencia na Tabela 01.

\section{DISCUSSÃO}

Esta pesquisa evidenciou a efetividade da implantação do protocolo de lesão por pressão, por meio da padronização de rotinas de trabalho nos serviços de saúde que ocorre conforme a necessidade dos eventos adversos e mudança de cultura de segurança, que deve ser sistematizada e num contexto linear15. Visto que a educação em saúde é um estratégia para implementação de protocolos que diminuam a barreira da realidade com um cenário ideal de diminuição de eventos adversos 16 .

Uma revisão integrativa da literatura com 15 artigos, no período de janeiro de 2018 a dezembro 2019, cujo cunho era identificar os fatores de risco para o desenvolvimento de LP em pacientes internados em Unidades de Terapia Intensiva demonstrou a importância da elaboração de protocolos institucionais, que incluam ações voltadas para a prevenção de LP, pois assim permite uma assistência de acordo com as necessidades e individualidade de cada paciente 17.

O hospital da pesquisa possui instituído o protocolo de prevenção de LP e reforça que o uso de protocolo para auxiliar na prevenção de lesão, foi considerado a ferramenta mais efetiva para diminuir o acometimento de incidentes, conforme o estudo realizado com 83 dados coletados da Comissão de Curativos do hospital escola Recife- PE, indo no mesmo seguimento deste estudo que se teve baixo índice de incidência de LP18.

A aplicação da Escala de Braden como instrumento de avaliação clínica do enfermeiro avalia os fatores favoráveis a lesionar a integridade da pele, para estabelecer fato-

\begin{tabular}{llll}
\hline Estádio da LP & Estágio I & 5 & 71 \\
& Estágio II & 2 & 29 \\
$\begin{array}{l}\text { Tempo de Internação } \\
\text { Hospitalar }\end{array}$ & Igual há cinco dias & 1 & 14 \\
& De 06 a 15 dias & 3 & 43 \\
Qucima de 16 dias & 3 & 43 \\
Quantidade da LP & Única lesão & 4 & 57 \\
Múltiplas lesões & 3 & 43
\end{tabular}

res que possam interferir na plenitude da pele, direcionar o enfermeiro a prescrever medidas preventivas e qualificar o serviço prestado19.

A revisão integrativa no período de 2006 a 2016 com 21 artigos, teve como objetivo identificar na literatura cientifica fatores predisponentes para o surgimento de LP em pacientes idosos, evidenciou-se que a senilidade traz uma susceptibilidade a incidência de lesões, decorrente do processo natural do envelhecimento, pois a pele perde em espessura, fibras elásticas, colágeno, perda de tecido adiposo e os capilares da derme que resulta em desidratação, tornando-a predisposta ao surgimento de injúria tecidual agravando ainda mais quando se constata mobilidade prejudicada20.

E o estudo realizado em hospital escola da cidade de Recife, feita com um pesquisado tipo transversal, retrospectivo, descritivo e de natureza quantitativa, com 1.234 participantes objetivou identificar o perfil epidemiológico dos pacientes acometidos por lesão, sendo que a faixa etária predominante foi acima de 60 anos (45,9\%). Estes dados corroboram com os encontrados neste estudo, visto que a prevalência de LP foi na população idosa21.os quais reforçam o estudo realizado através de análise dos registros em 160 prontuários do maior hospital público de atenção terciária do Estado do Ceará aonde chegaram à conclusão de que o índice em indivíduos com idade superior a 60 anos foi o maior entre a população analisada, chegando $54,4 \%$ da somatória total22.

No que tange a prevalência no sexo masculino vai de encontro com a pesquisa feita através de revisão interativa de literatura elaborada em cima de 07 artigos selecionados e retirados de base de dados eletrônicos, chegando à conclusão que $80 \%$ da amostragem de 07 artigos lidos na integra e apontou no sexo masculino com maior prevenção a desenvolver LP6. Já o estudo realizado num hospital do estado do Pernambuco, o qual analisou a amostragem de 1.234 casos notificados no decorrer dos anos de 2017 e 2018, e evidenciou-se o equilíbrio no surgimento da LP entre os gêneros, quando comparado entre os anos, onde tabulou 53\% dos casos de LP notificados, no ano de 2017 , sendo do gênero feminino e 53,97\% em 2018, acometeram o gênero masculino23.

Outro aspecto importante, inerente a suscetibilidade em adquirir lesões é o nível neurológico do indivíduo, já que algumas doenças crônicas interferem na mobilidade do paciente, fazendo com que passe mais tempo ao leito favorecendo a instalação de LP. Este estudo apresentou que da amostragem foi classificada com Glasgow leve, diferentemente de um estudo realizado no período de novembro de 2015 até outubro de 2016 no centro de terapia intensiva do hospital universitário do Sul do Brasil em que a maior porcentagem da população foi de Glasgow grave com escore $\leq 6$ representando $60,9 \%$ dos casos 24 .

Um estudo desenvolvido no Setor de Controle de Infecção (SCIH) de um hospital universitário público do interior do Paraná, apontou que a disfunção neurológica como a segunda patologia que torna o paciente mais suscetível a adquirir a LP, devido ao comprometimento da mobilidade do indivíduo, a disfunção neurológica ficou com $26 \%$ dentre as 227 fichas de notifica- 
ção de LP recebidas no período da pesquisa, ficando atrás apenas da traumatologia que somou $27,30 \% 25$. Fortalecendo a influência da presença de morbidades no surgimento de LP, o estudo realizado na unidade de acidente vascular cerebral (AVC) de um hospital universitário do Sul do Brasil, relata que o surgimento de LP está entre as maiores complicações clínicas sofridas pelo paciente acometido de AVC ou acidente isquêmico transitório (AIT) por afetar a mobilidade do paciente, percepção sensorial, circulação e oxigenação 26 .

Esse fator com índice elevado é justificado devido os indivíduos com algum tipo de doença crônica apresenta baixa imunidade, favorece as infecções e se agrava quando acometido por doenças neoplásicas a qual pode decorrer de deficiência nutricional proporcionando a instalação de LP com mais facilidade 27.Uma abordagem quantitativa, de caráter observacional analítica composta por 85 pacientes que estiveram internados em um hospital público de alta complexidade situado no interior do Estado da Paraíba, constatou que a hipertensão e a diabete estão entre as doenças crônicas que mais sobre saem em indivíduos acometidos por LP, sendo que 10 possuíam DM, 21 possuíam HAS e 33 possuíam as duas doenças 28.

A prevalência da lesão em grau I é contraditória ao estudo realizado através de coleta documental de um hospital de estudo da cidade de Curitiba - PR, que apontam a LP tissular profunda, com maior incidência contabilizando $37,5 \%$ dos 9 casos analisados, essa classificação é preocupante pois trata dano que pode ser evitado ou impedir a evolução da LP caso o enfermeiro identifique o paciente como de risco a desenvolver a LP29.

Em relação à localização mais acometida, prevalece a região sacra e com classificação de grau I e II, o que corrobora com um estudo realizado num hospital público do norte do estado de Santa Catarina, onde cita na região sacral como a mais acometida 17 (30,9\%), seguida da região occipital 12 $(21,8 \%)$ e calcâneos 11 (20\%), e as categorias mais prevalentes o nível I e II30. A região sacra também foi apontada como a localização que mais sofre com degeneração tecidual conforme pesquisa realizada em uma Unidade Terapia Intensiva de adultos de um hospital público das regióes Norte e Nordeste, por conta da anatomia a região recebe alta pressão devido posição em que o indivíduo acamado tende a ficar por mais tempo, ser decúbito dorsal31.

Outro aspecto que contribui fortemente para instalação de LP é o tempo de internação, quanto maior o período de hospitalização mais suscetível estará o paciente conforme dado na tabela que demonstra que $92 \%$ das LP surgiram em pacientes com internamento superior a 06 dias. Já o estudo realizado em cinco unidades de terapia intensiva de uma instituição hospitalar de porte especial situada a noroeste do estado de São Paulo no período de um ano sendo este de setembro de 2014 e agosto de 2015 onde observou-se que o tempo superior a 10 dias em UTI é um fator de alto teor para o desenvolvimento de LP27.

Quanto maior o tempo de exposição de proeminência óssea com a superfície mais o indivíduo se torna vulnerável a adquirir a LP, como ocorreu com 22 cadeirantes da Leme, que participaram de um estudo com objetivo compreender a interferência de LP na qualidade de vida do indivíduo e dos que os cercam, as principais queixas foram: a dependência de alguém para troca de curativo que resulta em limitação em seu cotidiano, medo da morte decorrente da lesão, odor exalados pelas lesões que muitas vezes acarreta no isolamento social devido o constrangimento diante do mau cheiro, todos agravantes citados reflete na sua vida social, familiar e do trabalho32.

A LP interfere diretamente na qualidade de vida do indivíduo e de sua família, tendo em vista primordial a prevenção, não conseguindo êxito na tentativa de evitar sua instalação, deve-se trabalhar com planejamento focando num tratamento individualizado com base em conhecimentos científico para resultar em uma reversão do quadro em menor tempo possível, para que se possa devolver a este paciente sua qualidade de vida 33

\section{CONCLUSÃO}

A existência de protocolos de segurança do paciente instituído no hospital é aliada na prestação da assistência, com objetivo de padronizar as condutas de enfermagem durante o período de internação dos participantes. Visto a atualização das ações em saúde com as tecnologias, resulta em baixa incidência de LP e qualidade de serviço. Contudo este estudo enfrentou as seguintes limitações que foi a subnotificação de incidência de LP no setor de qualidade e a falta de seguimento do preenchimento da escala de Braden durante o período de internação.

\section{Outro aspecto}

que contribui

fortemente para

instalação de

LP é o tempo de

internação, quanto

maior o período de

hospitalização mais

suscetível estará o

paciente conforme

dado na tabela que

demonstra que $\mathbf{9 2 \%}$

das LP surgiram

em pacientes com

internamento

superior a 06 dias 


\section{REFERÊNCIAS}

1. Molinari J, Luna, MMM. 0 processo de certificação de qualidade e acreditação hospitalar uma revisão da literatura. XXVII Simpósio de Engenharia de Produção Bauru [internet]. 2020 nov [cited 2021 set 12] Available from: https://www.researchgate.net/profile/Monica-Luna-8/publication/348048989.

2. Sampaio LRL, Carvalho TB, Sampaio BBL, Fernandes MNM, Oliveira CC de. Prevenção de lesão por pressão: conhecimento e ações de cuidadores e pacientes domiciliares [Internet]. 2019 Dez. [cited 2021 out 30] Journal Health NPEPS. Available from: https://periodicos.unemat.br/index.php/jhnpeps/article/view/3981

3. Alves NF, Salome GM, Aplicativo "SICKSEG" Em Plataforma Móvel Para a Prevenção de lesões cutâneas. [internet]. 2020 abr. 14. [cited 2021 out 30] Revista de Enfermagem UFPE. DOI: 10.5205/19818963.2020.244152 Available from: https://periodicos.ufpe.br/revist as/revistaenfermagem.

4. Ministério da Saúde (BR). RDC N 36, de 25 de julho de 2013. [Internet]. 2013 [acesso 15 de março de 2020]; Direção eletrônica: https://bvsms.saude.gov.br/bvs/saudelegis/anvisa/2013/ rdc0036_25_07_2013.html.

5. Ribeiro AMN, Ribeiro EKC, Ferreira MTA, Sousa JERB, Silva AAS, Baldoino LS. Conhecimento de acadêmicos de enfermagem sobre lesão por pressão [Internet]. 2019 jul[cited 2021 ago. 01]. Revista Rene. Available from: DOI: 10.15253/2175-6783.20192041016

6. Alencar GSA,SilvaMN,AssisAV,SouzaMNA,PereiraJLF,Oliveira WB, et al. Lesão por pressão na unidade de terapia intensiva: incidência e fatores de riscos[Internet]. 2018 fev[cited 2021 ago 01]. Revista Nursing. Available from: DOI: http://www.revistanursing.com.br/ revistas/239-Abril2018/lesao_por_pressao_na_unidade_de_terapia_intensiva.pdf

7. Jesus MAP, Pires OS, Biondo SC, Matos RM. Incidência de lesão por pressão em pacientes internados e fatores de risco associados [Internet]. 2020 [cited 2021 ago.01]. Revista Baiana Enfermagem. V.34. Available from: DOI: http://dx.doi.org/10.18471/rbe.v34.36587

8. Feitosa DVS, Silva NOS, Pereira FNM, AlmeidaTF, Estevam AS. Atuação do enfermeiro na prevenção de lesão por pressão: uma revisão integrativa da literatura [Internet]. 2020 mar [cited 2021 ago. 01]. Revista Eletrônica Acervo Saúde. Vol.n.43. Available from: DOI: https://doi.org/10.25248/reas.e2553.2020

9. Amaral APS, Pinheiro GML. Reflexões sobre os protocolos do Programa Nacional de Segurança do Paciente em um hospital público [Internet]. 2021 Jan [cited 2021 ago. 01]. Lecturas: Educación Física y Deportes. Available from: https://doi.org/10.46642/efd.v25i272.908

10. Lima RMLS, Moura MV, Matos JC, Walter KC, Oliveira ACGDPC, Araújo VS, et al. Conhecimento dos enfermeiros acerca da importância do uso de protocolos de cuidados: Discurso do sujeito coletivo[Internet]. 2021 [cited 2021 ago 01]. Research, Society and Development, V.10, n.1. Available from: DOI: 10.33448/rsd-v10i1.11186

11. Holanda OQ, Oliveira VA, Fernandes FECV ,Xavier SB, Mola, R. Efetividade do protocolo para prevenção de lesões por pressão implantado em unidade de terapia intensiva[Internet]. $2018 \mathrm{dez}$ [cited 2021 ago. 01]. Revista Espaço para a Saúde. Available from: DOI: 10.22421/15177130-2018v19n2p64

12. Mutou FML, Nascimento KM, Monari C,Santos AP, Silva LR, Neto JSA, et al. Lesões por pressão: a gestão de saúde do ponto de vista multiprofissional[Internet]. 201 ago [cited 2021 ago 01]. Revista
Cientifica.V.4,n.2. Available from: http://seer.umc.br/index.php/revistaumc/article/view/617/739

13. Portugal LBA,Christovam BP. Estimativa do custo do tratamento da lesão por pressão, como prevenir e economizar recursos [Internet]. 2018 [cited 2021 ago. 01]. Revista de Enfermagem Atual. Available from: https://revistaenfermagematual.com.br/index.php/ revista/article/view/93/18

14. Andrade CCD, Almeida CFSC, Pereira WE ,Alemão MM, Brandão CMR, Borges EL. Custos do tratamento tópico de pacientes com úlcera por pressão [Internet]. 2016 mar [cited 2021 ago. 01]. Revista da Escola de Enfermagem da USP. Available from: DOI: http://dx.doi. org/10.1590/S0080-623420160000200016

15. Santos ESS, Silva GER, Pinheiro AKC, Gomes BSG, Oliveira APR, Rodrigues JP, Matos WDV, et al. Pratica de gestão e gerenciamento em enfermagem: uma experiência sobre o Processo de Acreditação Hospitalar em Belém-PA. Anais do $13^{\circ}$ Congresso Internacional da Rede Unida [Internet]. 2018 jan.[cited 2021 ago. 01]. Available from: http://conferencia2018.redeunida.org.br/ocs2/index. php/13CRU/13CRU/paper/view/3209/0

16. Macedo, A. B. T., Mello, D. B., Graciotto, A., Cortelini, C. L., Souza, E., Picetti, N. Lesões por pressão em adultos portadores de germes multirresistentes: um estudo de coorte. Revista Saúde Coletiva Barueri. 2021; [cited 2021 nov 09] (11) N.69; 8348. Available from: https:/doi.org/10.36489/saudecoletiva.2021 v11 i69p7000

17. Ferro ZLA, Rios RAS, Santos CJC, Pereira R, Rocha LCSL, Almeida HFR. Fatores de risco para lesão por pressão em unidades de terapia intensiva: uma revisão integrativa da literatura [Internet]. 2020 [cited 2021 ago 01]. Brazilian Journal of health Review. Available from:DOI:10.34119/bjhrv3n5-116

18. Barretos RMS, Santos RLN, Melo EMVB. Perfil de pessoas com lesão por pressão internados na unidade de terapia intensiva [Internet]. 2019 dez [cited 2021 ago. 01]. Revista O Mundo da Saúde. V.43 n.4. Available from: DOI: 10.15343/0104-7809.2019430410301043

19. Machado LCLR, Fontes FLL, Sousa JERB, Neta ASS, Alencar EJC, Costa ACRR, et al. Fatores de risco e prevenção de lesão por pressão: aplicabilidade da Escala de Braden [Internet]. 2019 mar [cited 2021 ago. 01]. Revista Eletrônica Acervo Saúde. V.21. Available from: https://doi.org/10.25248/reas.e635.2019

20. Souza NR, Freire DA, Souza MAO, Melo JTS, Santos LV, Bushatsky M. Fatores predisponentes Para o Desenvolvimento da Lesão por Pressão em Pacientes Idosos: Uma Revisão Integrativa [Internet]. 2017 [cited 2021 ago 01]. Revista ESTIMA. V.15, n.4, pag.229-239. Available from: DOI: 10.5327/Z1806-3144201700040007

21. Melo DPL, Moura SRS, Rocha GMS. A prevalência de lesão por pressão em um hospital escola [Internet]. 2020 nov [cited 2021 ago. 01]. Revista Cientifica de Enfermagem. V.11 n.33. Available from: DOI: https://doi.org/10.24276/rrecien2021.11.33.27-34

22. Barbosa A, Oliveira E, Leite M, Feitosa DS, Studart RM, Cavalcante T de M, Oliveira PM, et al. Perfil clínico dos pacientes acometidos por lesão por pressão [Internet] ago2019 [cited 2021 ago. 01]. Available from: https:/www.revistaenfermagematual.com.br/index.php/ revista/article/view/161

23. Moura SRS, Melo DPL, Rocha GMS, Cruz ERC. Prevalência de lesão por pressão em um hospital geral [Internet]. 2020 out [cited 2021 ago. 01]. Revista Eletrônica Acervo Saúde. Available from: 


\section{artigo}

Santos, A. R., Moura, V. L. L., Koller, F. J., Batista, J., Burdzinski, V. F., Oliveira, A. A. S.

Incidência de lesão por pressão em pacientes internados em um hospital com certificação de qualidade

\section{REFERÊNCIAS}

https://doi.org/10.25248/reas.e4298.2020

24. Lopes ANM, Batassini E, Beghetto MG. Lesão por pressão em uma coorte de pacientes críticos: incidência e fatores associados [Internet]. 2021 [cited 2021 ago. 01]. Revista Gaúcha Enfermagem. Available from: doi: https://doi.org/10.1590/1983-1447.2021.20200001

25. Tauffer J. Alves DCl, Zack BT, Berticelli MC, Kassim MJN, Carmello SKM.et al. Perfil epidemiológico das lesões por pressão em um hospital escola no Oeste do Paraná[Internet]. 2019 nov [cited 2021 ago 01]. Rev. Adm. Saúde. V19. Available from: http://dx.doi. org/10.23973/ras.77.189

26. Gaspari AP, Cruz EDA, Batista J, Alpendre FT, Zétola V, Lange MC. Preditores de internação prolongada em Unidade de Acidente Vascular Cerebral (AVC). [Internet]. 2021 out [cited 2021 out 15]. Rev. Latino-Am. Enfermagem. Available from: DOI: http://dx.doi. org/10.1590/1518-8345.3118.3197.

27. Pachá HHP, Faria JIL, Oliveira K A, Beccaria LM. Lesão por Pressão em Unidade de Terapia Intensiva: estudo de caso-controle[Internet]. 2018 nov [cited 2021 ago. 01]. Revista Brasileira de Enfermagem. vol.71 no.6. Available from: https://doi.org/10.1590/0034-71672017-0950

28. Rocha SS, Falcone APM, Pontes EDS, Rocha SRS. Análise da presença de lesão por pressão em pacientes hospitalizados e as principais comorbidades associadas [Internet]. 2020 mar [cited 2021 ago 01] Research, Society and Development v. 9, n. 4. Available from: DOI: $10.33448 /$ rsd-v9i4.3009

29. Leites AWR, Almeida TQR, Arué AM, Ribeiro GPR, Danski VRR.
Reichembach T. Lesão por pressão em pacientes adultos internados e atendidos por um serviço especializado da pele no maior hospital do Paraná [Internet]. 2020 [cited 2021 ago 01] Research Society and Development, v. 9. Available from: DOI: http://dx.doi.org/10.33448/ rsd-v9i9.6924

30. Otto C, Schumacher B, Wiese LPL, Ferro C, Rodrigues RA. Fatores de risco para o desenvolvimento de lesão por pressão em pacientes críticos [Internet]. 2019 [cited 2021 ago 01] Revista Enferm Foco. Available from: http://biblioteca.cofen.gov.br/wp-content/uploads/2019/03/Fatores-De-Risco-Para-o-Desenvolvimento-DeLes\%C3\%A3o-Por-Press\%C3\%A3o-Em-Pacientes-Cr\%C3\%ADticos. pdf

31. Teixeira AKS. Nascimento TSN. Sousa TL, Sampaio LRL, Pinheiro ARM. Incidência de lesões por pressão em Unidade de Terapia Intensiva em hospital com acreditação [Internet]. 2017 nov [cited 2021 ago 01] Revista ESTIMA, v.15. Available from: DOI: ttps://doi. org/10.5327/Z1806-3144201700030006

32. Ferro BH, Renner JS, Barth M, Manfio EF. A influência das lesões por pressão na qualidade de vida e inclusão são social: a percepção dos usuários de cadeiras de rodas[Internet]. 2020 abr.[cited 2021 ago. 01] Revista Interdisciplinar de Estudos em Saúde, v.9. Available from: DOI: https://doi.org/10.33362/ries.v9i1.1739

33. Jaques DF, Silva SF, Santos $A A$, Resende MA, Calsavara RA, Barros $\mathrm{PA}$, et al. A prevenção de lesão por pressão em pacientes acompanhados pelo enfermeiro da estratégia de saúde da família[Internet]. 2020 jun. [cited 2021 ago 01] REAS/EJCH. Vol.Sup.n.50. e2313. Available from: DOI: https://doi.org/10.25248/reas.e2313.2020 